\title{
Congenital Long QT Syndrome in an Infant
}

\author{
Aris Lacis, Inga Lace, Elina Teivane, Vita Knauere, Inguna Lubaua, \\ Inta Bergmane, Valts Ozolins, Lauris Smits, Normunds Sikora \\ University Hospital for Children, Riga, Latvia \\ Department for Pediatric Cardiology and Cardiac Surgery
}

\section{Summary}

Long QT syndrome (LQTS) is a disorder of myocardial repolarization characterized by prolonged QT interval on ECG with prevalence close to $1 / 3000-1 / 5000$. LQTS is characterized by the occurrence of syncopal episodes due to torsades de pointes ventricular tachycardia (VT) and by a high risk for sudden cardiac death among untreated patients $(1,2,3)$. In 12\% of patients with LQTS, sudden death is the first manifestation of the disease and only in $4 \%$ this happens in the first year of life (2). There is consensus that all symptomatic children with LQTS should be treated with $\beta$-blockers which are effective in preventing cardiac events and reducing mortality in $70 \%$, but do not protect patients from sudden death completely $(1,2,3,4)$. The prognosis is poor in untreated patients with annual mortality $20 \%$ and 10 year mortality up to $50 \%(1,2)$. Here we present a case of relatively rare congenital heart rhythm disorders in an infant which required immediate treatment.

Key words: long QT syndrome; congenital arrhythmia.

\section{AIM OF THE DEMONSTRATION}

We present a case of congenital heart rhythm disorders in an infant which carry serious risk of sudden death if left untreated.

\section{CASE REPORT}

The child was sent to the hospital at the age of 21 days by the general practitioner due to bradycardia detected within a routine visit. The girl was born from the first pregnancy in urgent cesarean section due to fetal distress (umbilical cord wrapped around the neck) at 40 weeks of gestation, birth weight $3090 \mathrm{~g}$, height $54 \mathrm{~cm}$, Apgar score 7/9 and was discharged from the maternity hospital at the age of 4 days. The child received breastfeeding and parents did not have any complains about the newborn. The mother of the child is 21 years old and the father 23 years old, both completely healthy. The course of the pregnancy was uneventful. There were no cases of heart rhythm disorders or sudden death in the family history, no chronic illnesses in the family history. At the time of hospitalization (at the age of 21 days) the weight of the child was $3265 \mathrm{~g}$, bradycardia-heart rate 72 times per minute was detected, no heart murmurs, no hepatomegaly. Repeated electrocardiograms (ECG) showed first to second degree second type ( Mobiz II) atrio-ventricular block and prolonged QT interval 0,52, cQT 0,54 as well as complete right and left anterior bundle branch block. Echocardiography was completely normal with LVDd $22 \mathrm{~mm}$, fractional shortening $37 \%$ and ejection fraction $61 \% .24$ hour ECG monitoring reveled first to second degree second type ( Mobiz II) atrio-ventricular block and prolonged QT interval and repeated paroxysms of ventricular tachycardia with the ventricular rate up to 251 beats per minute ( the longest one 49 seconds). No changes were found in thoracic x-ray, neurosonography and abdominal ultrasound. There were neither signs of acute infections nor TORCH infections present. There were no positive auto antibodies detected. At the age of 25 days epicardial pacemaker (DDD regimen) was implanted in the abdominal front wall and therapy with $\beta$-adrenoblocker Propranolol started. The postoperative period was uneventful and the girl was discharged at the seventh day after the operation. No prolongation of cQT was observed in family members of the girl. The hearing was detected to be normal. The analyses to detect the genetic background showed mutation characteristic for LQTS type 2 (mutation in gene HERG on chromosome7). The age of the girl is seven months now, she is followed up by pediatric cardiologist, receives Propranolol, no serious hearth rhythm disorders observed. The pacemaker regimen is DDD ( lowest rate 90 beats per minute, upper track 200, AS-VS 69\%, AS-VP 29\%, APVS $1,5 \%$, AP-VP 0,2\%, threshold impedance A $0,375 \mathrm{~V}$,

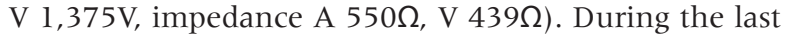
check up the pacemaker diagnostic data and 24 hour ECG monitoring showed no runs of tachycardia.

\section{DISCUSSION}

QT prolongation may be congenital or acquired. Long QT syndrome is a genetic disease due to mutations of several genes all encoding ionic (potassium or sodium) channels involved in the control of ventricular repolarization. The characteristic ECG shows a prolonged QT interval (usually cQT greater than 0,46 seconds), abnormal Twave morphology (bifid, biphasic or notched), $20 \%$ bradicardia due to second degree AV block, monomorphic or polymorphic ventricular tachycardia( present in 10$20 \%)(1,2,3)$. Based on genetic background, six types of Romano-Ward syndrome (LQTS 1-6, autosomal dominant, with normal hearing) and two types of Jervell and Lange-Nielsen syndrome (JLN 1-2, autosomal recessive, with deafness) are identified. Two additional syndromes (Andersen-Tawil and Timothy syndrome are 
considered different subgroups $(1,3)$. In our case there is evidence of characteristic genetic changes of LQTS 2. The LQT2 type is the second most common gene location that is affected in long QT syndrome, making up about 35 to 45 percent of all cases. This form of long QT syndrome most likely involves mutations of the human ether-a-go-go related gene (HERG) on chromosome 7 . The HERG gene (also known as KCNH2) is part of the rapid component of the potassium rectifying current $\left(\mathrm{I}_{\mathrm{Kr}}\right)$. (The $\mathrm{I}_{\mathrm{Kr}}$ current is mainly responsible for the termination of the cardiac action potential, and therefore the length of the QT interval.) The normally functioning HERG gene allows protection against early after depolarizations ( 1 , $3,4)$. In most cases, several members of the same family are gene-carriers (family history positive in about 60\% of cases). Low penetrance exists in LQTS, which means that gene-carriers may not show the clinical phenotype and may have a normal QT interval (2). Therefore a normal QT in the parents does not rule out familial LQTS. In addition, approximately $30 \%$ of cases are due to 'de novo' mutations which imply unaffected parents and no family history. 'De novo' LQTS mutations have been demonstrated in infant victims of cardiac arrest and sudden death diagnosed as Sudden Infant Death Syndrome $(1,2)$. However, about $40 \%$ of the families with LQTS have not yet been linked to any of the known genes. Our patient showed serious hearth rhythm disorders already during the neonatal period. Relatively few LQTS patients have cardiac events during the first year of life, the vast majority become symptomatic later on, either during childhood or adolescence according to genetic subgroups $(1,2,3)$. The acquired causes of LQT (including drugs, electrolyte imbalance, marked bradycardia, cocaine, organophosphorus compounds, subarachnoid hemorrhage, myocardial ischemia, protein sparing fasting, autonomic neuropathy, and human immunodeficiency virus disease) were excluded. Betablockers are the first choice therapy in LQTS and are effective in preventing recurrences in $80 \%$ of already symptomatic patients; different degrees of protection exist according to genetic subgroups. If beta-blockers are unable to prevent new cardiac events, additional drug therapy, left cardiac sympathetic denervation, pacemakers or the implantable cardioverter defibrillator should be considered based on evidence, with consideration for body size $(1,2,3,4)$. In our case there were indications for both implantable pacemaker and the therapy with beta-blockers. Despite this combined therapy there is still risk for serious heart rhythm disorders but the risk for sudden death would be much higher if left untreated. With a prevalence 1/3000$1 / 5000$ and a manifestation within the first month of life this case is a rarity in our small population but in the case of pathology with potentially life threatening consequences it is very important to diagnose it as early as possible.

\section{Conflict of interest: None}

\section{REFERENCES}

1. Allen HD, Driscoll DJ, Shaddy RE, Feltes TF. Disorders of Cardiac Rhythm and Conduction // In: Heart Disease in Infants, Children, and Adolescents. 7th Edition, USA: Moss and Adams, Lippincott Williams and Wilkins; 2008,Vol1, $328-331$

2. Khan, Ijaz A. Long QT Syndrome: Diagnosis and Management // Am Heart J, 2002; 143(1):7 - 14

3. Park MK, Cardiac Arrhythmias // in: Pediatric Cardiology for Practitioners, 5th Edition, USA: Mosby Elsevier; 2008; 437 - 443

4. Schwartz PJ, Garson A, Paul T, et al. Guidelines for the interpretation of the neonatal electrocardiogram // Eur Heart J, 2002; 33:1329 - 1344

\section{Address:}

Elina Teivane

Children`s Clinical University Hospital,

Department for Pediatric Cardiology and Cardiac

Surgery

Vienibas gatve 45, LV-1004,

Riga, Latvia

E-mail: eteivane@inbox.lv
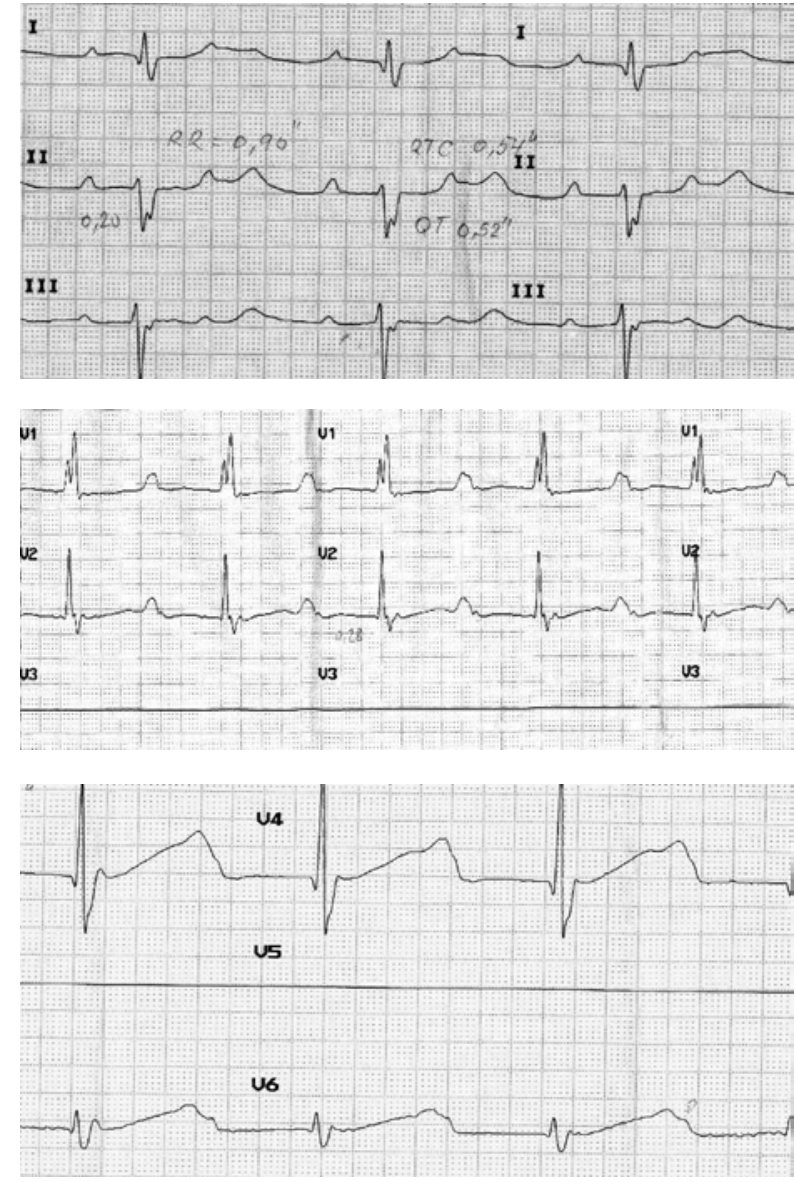

The ECG of the 24 days old infant shows prolongation of cQT $>500 \mathrm{~ms}$ and atrio-ventricular block first to second degree Mobitz II 